\title{
Identification of gallstone samples by laser-induced fluorescence
}

\author{
Andrew L. Fanai, S. Rai \\ Department of Physics, Mizoram University, Tanhril 796004, Mizoram, India \\ Corresponding author: srai.rai677@gmail.com
}

\begin{abstract}
Laser-induced fluorescence spectra of gallstone samples from three different age group was analysed in the visible region. The gallstones were obtained from patients at Aizawl Civil Hospital, Mizoram, Northeast India, who had undergone surgery. Subjects included both adults and children patients so that the age group was classified as group A consisting of $\mathbf{3 0}$ years, group B consiting of 39 years, and group C consisting of 60 years of age. The stones were washed with deionized water and dried. The absorption spectra for all the mixed type gallstone samples show only one broad band in the visible region that lies in the 400-800 nm region with $\lambda_{\max }$ at about $450 \mathrm{~nm}$. Significant changes were observed when different solvents were used. Our present understanding is that the most important mechanism whereby cholesterol is held in solution in the bile is that it is taken into miceller solution in combination with phospholipids and bile salts.
\end{abstract}

Keywords: Laser-induced fluorescence spectra, gallstone, visible spectrum, solvents.

\section{INTRODUCTION}

The gall bladder is a small muscular pear-shaped digestive storage organ that lies beneath the right lobe of the liver. Its function is to store and slowly release bile into the digestive system for digestion of the fats. With the intake of fats, both the liver and the gall-bladder excrete bile to the stomach for digestion. Once digestion stops and if the gallbladder is not emptied out completely the bile that remains in the gall bladder can become too concentrated with cholesterol, and as a result gallstones are formed. This is a common disease and constitutes a major health problem worldwide. It is of considerable interest to biochemists and biophysicists due to the complicated reactions involved. These deposits have a large fraction of organic substances. Other important constituents are bilirubin, fatty acids and minerals like aragonite, calcite, valerite, opatite, calcium palmitate, with various trace elements like chromium, iron, copper and magnesium. Gallstones can be conveniently grouped into four major types depending on their chemical constituents.

There are three major type of gallstones found in patients, white, black and brown. The major chemical components of white stones are cholesterol. They are (a) mixed stones which consist of varying concentrations of bilirubate salts and cholesterol; (b) cholesterol stones containing $>70 \%$ cholesterol by weight; (c) brown pigment stones; and (d) black pigment stones. Brown and black pigment stones both consist mainly of different salts of bilirubin with less than $10 \%$ cholesterol by weight. Gallstone occurrence is reported to be higher in western countries where cholesterol and mixed pigment stones predominate (Ishida et al., 1987,). The occurrence of both groups of stones is 2-3 times more common in women than men. The mixed stones are the most common variety of gallstones. Within this category, considerable differences in size, color, number, and chemical composition can occur. These types of stones often exhibit concentric layers of cholesterol and pigment like the rings in a tree trunk. The nature of the center of nucleus can also vary greatly. Crystals of cholesterol, calcium and pigment salts are commonly found. Pure cholesterol gallstones are rare, often solitaire and constitute about 6$10 \%$ of stones in developed countries. They are round and have light colored crystalline appearance. Brown pigment stones are rare in Western countries. They are soft and crumble easily. Black pigment stones constitute up to $20 \%$ of gallstones in the Western countries. They vary in size, number and hardness. Since the process of 
formation of gallstones involves a long time it is not completely understood. It is known that one essential prerequisite in their formation is a supersaturated cholesterol environment together with nucleating factor.

The identification of the components of gallstone is essential as it provides information that could be useful for medical practioner to find out the underlying cause of gallstones and to decide whether to treat gallstone patients surgically or therapeutically. The composition of gallstones as well as their microstructures including the distribution of constituents within the stones needs to be determined in order to explain the formation. At present a number of methods of identifying gallstones exist. The usual method involve procedures to crush the stones into small particles, extraction and wet techniques which are tedious, time consuming and destructive. Conventional scanning electron microscopy, energy dispersive $\mathrm{X}$-ray methodology has been used to study the microstructure of gallstones. X-ray micro analysis allows the identification of calcium salts within the gallstone microstructure. Various calcium salts have been identified in the cores of both cholesterol and pigment stones and it has been suggested that calcium plays a role in the nucleation process. Infrared spectroscopy, FTIR, photoacoustic spectroscopy (PAS) are becoming routinely available as analytical tools. Detection of solid materials in some kidney and gallstones are reported to have been made by Raman spectroscopy (Rastogi et al., 1999). Recently, vibrational spectroscopic techniques for the characterization of some human gallstones have been identifying all the gallstone components and therefore a quick non destructive or minimally destructive procedure is yet to be realized. In the present work we have investigated the Laser-induced Fluorescence (LIF) \& FTIR spectra of a number of human gallstones of different types ( Rai et al., 1995; Rai et al., 2001)

\section{EXPERIMENTAL}

Gallstones were obtained from patients from the north east region of India undergoing surgery at Civil Hospital Aizawl from both adults and children patients for this study. The stones were washed with deionized water and dried. Three speimens for different age group are classified as A, B and C. The age group A belong to the age group 30 years. Group $B$ belongs to 39 years and C 60 years. The spectra were recorded bi iHR320 imaging spectrometer.

\section{RESUlTS}

\section{Absorption spectra}

The absorption spectra for all the mixed type gallstone samples show only one broad band in the visible region lies in the $400-800 \mathrm{~nm}$ region with $\lambda_{\max }$ at about
$450 \mathrm{~nm}$ (Figure 1). Significant changes are observed when different solvent are used. Our present understanding is that the most important mechanism whereby cholesterol is held in solution in the bile is that it is taken into miceller solution in combination with phospholipids and bile salts. Cholesterol, phospholipids and bile salts have both water soluble hydrophilic groups and water insoluble hydrophobic groups.

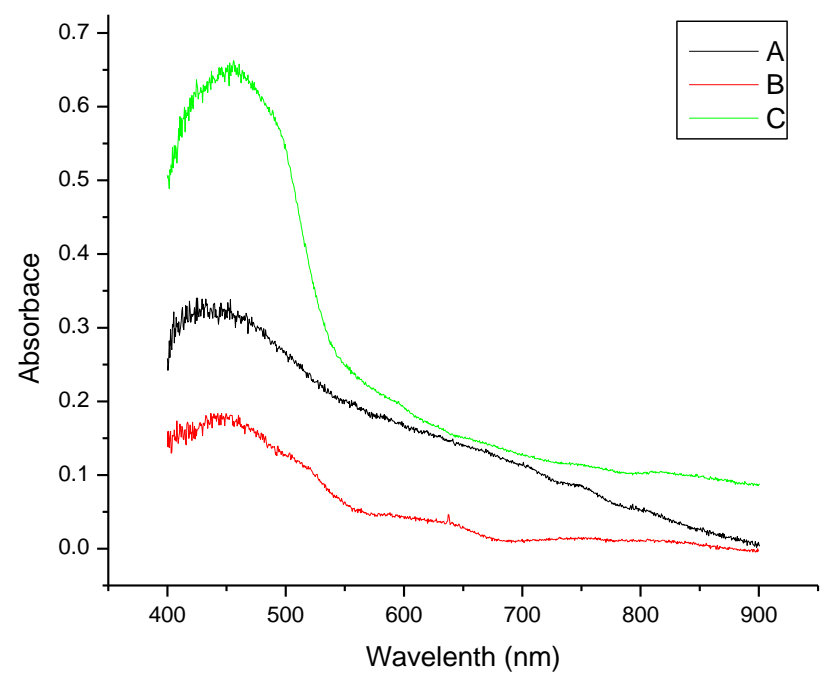

Figure 1: The absorption spectra of a specimen of three age group (A) 30 years, (B) 39 years (C) 60 years.

\section{Laser-induced fluorescence}

The fluorescence spectra as shown in Figure 2 for three different age groups are classified in A, B and C. Group A of age 30 years, group $B$ belong to age 39 years and group $C$ belongs to 60 years. The laser-induced fluorescence spectra for three different age groups range from 400-800 $\mathrm{nm}$. For lower age group four peaks appears at $517,529,650$ and $706 \mathrm{~nm}$ where the intensity $517 \mathrm{~nm}$ and $706 \mathrm{~nm}$ peaks are weaker than 529 and 650 $\mathrm{nm}$ peaks. The middle age group appears almost the same wavelength accept some peaks are shifted to higher wavelengths. For higher age group peaks appears at 517, 529, 658 and $711 \mathrm{~nm}$, the peaks for higher age group the peaks at 658 and $711 \mathrm{~nm}$ are comparatively at higher wavelengths.The fluorescence which is due to the bilirubin present in the mixed gallstone sample is observed in the 400-800 $\mathrm{nm}$. Laser-induced breakdown spectroscopy (LIBS) is one of the most appropriate analytical tools to get information about the constituents of gallstones. A LIB is based on collection of the emission from ionic, atomic and molecular species in the plasma formed due to the interaction of the high power laser and material as shown in the earlier paper (Pathak et al., 2011). 


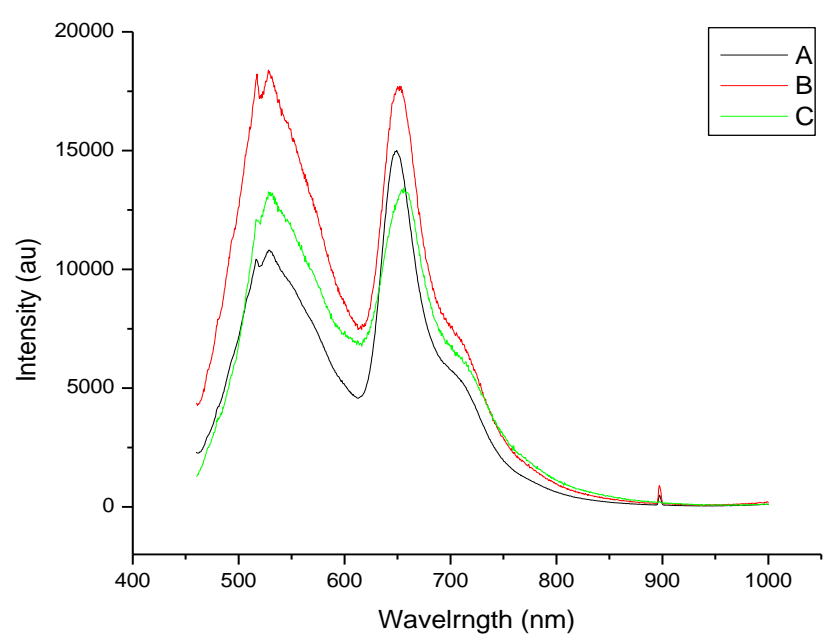

Figure 2: LIF of the specimens of gallstones (A) 30 years (B) 39 years (C) 60 years.

\section{FTIR spectroscopy of whitish brown gallstones}

The FTIR spectra of whitish brown gallstones are shown in Figure 3. The gallstones which contained more than $80 \%$ cholesterol were classified as pure cholesterol gallstones. Pure cholesterol gallstones were characterized by bands around 2936, 1464 and $1052 \mathrm{~cm}^{-1}$. The spectra of whitish brown of gallstones showed the presence of higher cholesterol content which is evident by the higher absorbance was characterized in the region between $2880-3000 \mathrm{~cm}^{-1}$ due to stretching vibrations of $\mathrm{CH}_{2}$ and $\mathrm{CH}_{3}$ groups and lower absorbance in the regions $1500-1700 \mathrm{~cm}^{-1}$ for bilirubin.

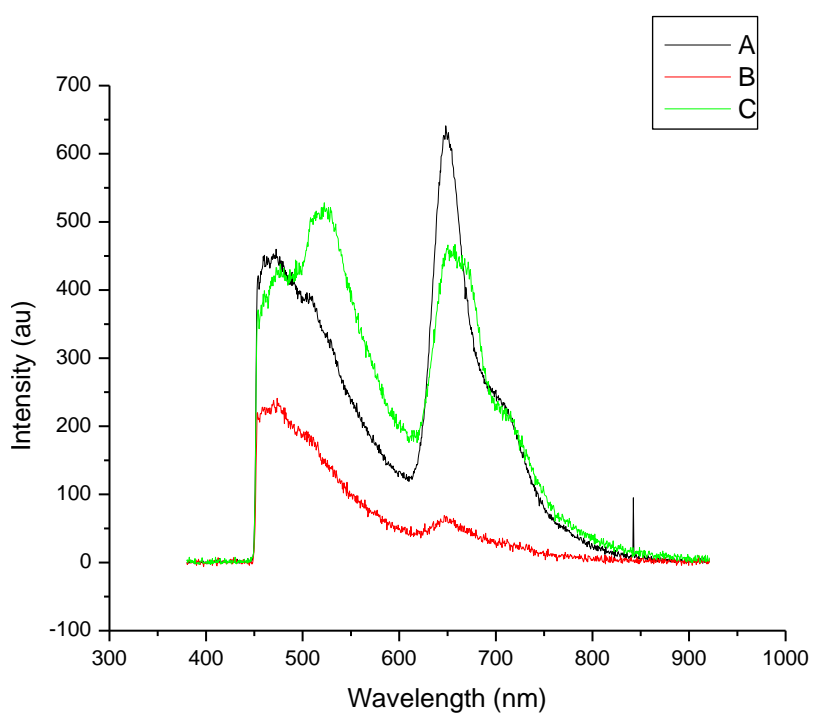

Figure 3: The FTIR spectra of whitish brown gallstone.

\section{DISCUSSION}

In conclusion, we have presented the result of absorption photoluminescence and FTIR spectra of some gallstones of different age groups, which indicates future perspective of these techniques for medical and biological purposes. The presence of hydrogen binding was observed in the IR spectrum which could be the hydrophilic group of the bile salts. The cholesterol is a typical and important steroid which contains diene systems. The conjugated diens shows absorption spectra in the ultra violet region in ethanol. It is worthwhile to indicate the LIF also indicate the effect of age. The fluorescence peaks appearing at $650 \mathrm{~nm}$ is very strong for all the age group. However it is difficult to record the Raman spectra of mixed gallstones due to their strong fluorescence. LIBS studies shows that the gallstone collected from NE India are particularly reach in the minerals calcium, magnesium, copper iron, silicon, sodium and potassium. The particular mineral composition of the gallstones may reflect the dietary habits.

\section{REFERENCES}

Baruah, G.D., Rai, S., Debnath, R.K. (1996). Laser-induced fluorescence and effect of gamma ray radiation on the IR, visible and UV absorption spectra of some mixed gallstones. Asian. J. Phy. 5(4), 399-403.

Ishida, H., Kamoto, R., Uchida, S., Ishitani, A., Ozaki, Y., Iriyama, K., Tsukie, E., Shibata, K., Ishihara, F., K a meda, H. (1987). Raman microprobe and Fourier transform infrared microsampling studies of the microstructure of gallstones. J Applied Spectroscopy. 41, 407-412.

Pathak, A.K., Rai, N.K., Rai, A.K., Rai, P.k., Rai, S., Baruah, G.D. (2011). Study of Different concentric rings inside gallstones with LIBS. Laser. M ed. Sci. 26, 531-537.

Rai, S., Debnath, R.K., Baruah, G.D. (1995). Laser-induced fluorescence of some mixed gallstones. Proceedings of National Laser Symposium, I.R.D.E. Dehradun. 10 February 2014, pp. 294-295.

Rai, S., Baruah, G.D. (2001). Identification of gallstones by laser-induced fluorescence Technique. Laser Horizon. 5, 14-16.

Rastogi, V.K., Tamuli, R., Rai, s., Pradhan, A., Bhattacharya, S., Baruah, G.B. (1999). $8^{\text {th }}$ European Conference on the Spectroscopy of Biological Molecules, 29 August-2 September 1999, Enschede, The Netharlands, pp. 627-229.

Sikkandar, S., Jayakumar, S., Gunasekaran, S., Renugadevi, T.S., Alwar, B. (2011). Study on the analysis of human gallstones using Fourier transform infrared spectroscopic technique. Int. J. Chem. Tech. Research, 3(1), 149-154. 